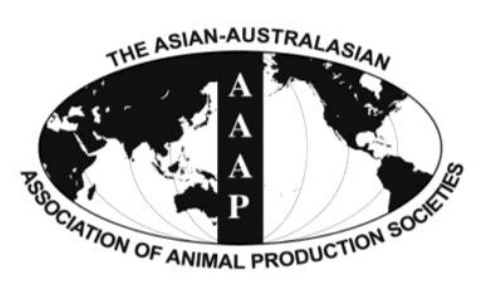

\title{
Antibacterial Activity of Recombinant Pig Intestinal Parasite Cecropin P4 Peptide Secreted from Pichia pastoris
}

\author{
Ki-Duk Song and Woon-Kyu Lee ${ }^{1, *}$ \\ Genomic Informatics Center, Han Kyong National University, Anseong 456-749, Korea
}

\begin{abstract}
Cecropins (Cec) are antibacterial peptides and their expression is induced in a pig intestinal parasite Ascaris suum by bacterial infection. To explore the usefulness of its activity as an antibiotic, CecP4 cDNA was prepared and cloned into the pPICZ B expression vector and followed by the integration into AOX1 locus in Pichia pastoris. The supernatants from cell culture were collected after methanol induction and concentrated for the test of antimicrobial activity. The recombinant $P$. patoris having CecP4 showed antimicrobial activity when tested against Staphyllococcus aureus in disc diffusion assay. We selected one of the CecP4 clones (CecP4-2) and performed further studies with it. The growth of recombinant P. pastoris was optimized using various concentration of methanol, and it was found that $2 \%$ methanol in the culture induced more antibacterial activity, compared to $1 \%$ methanol. We extended the test of antimicrobial activity by applying the concentrated supernatant of CecP4 culture to Pseudomonas aeruginosa and E. coli respectively. Recombinant $\mathrm{CecP} 4$ also showed antimicrobial activity against both Pseudomona and E. coli, suggesting the broad spectrum of its antimicrobial activity. After improvements for the scale-up, it will be feasible to use recombinant CecP4 for supplementation to the feed to control microbial infections in young animals, such as piglets. (Key Words: Cecropin, Ascars suum, Pichia pastoris, Methanol, Antimicrobial Activity)
\end{abstract}

\section{INTRODUCTION}

Antibiotics have been used for treating infectious diseases, preventing diseases, and for livestock and poultry production as animal growth promoter (AGP) (Allen et al., 2013). But the concerns about the increase in the incidence of human infections by antibiotic-resistant bacteria and their spread led to bans on the use of the antibiotics as AGP for livestock production in Korea and European nations as well (Allen et al., 2013). Nonetheless, the use of antibiotics is allowed only for treatment purpose of prevention, control, and treatment of specific animal diseases. The bans on the use of antibiotics encourages the development of alternatives to antibiotics, including antimicrobial peptides (AMPs), probiotics, organic acids and vaccines, for food-

\footnotetext{
* Corresponding Author: Woon-Kyu Lee. Tel: +82-32-890-0946, Fax: +82-32-890-1199, E-mail: wklee@inha.ac.kr

1 Laboratory of Developmental Genetics, Department of Advanced Biomedical Sciences, School of Medicine, Inha University, Incheon 400-712, Korea.

Submitted Sept. 30, 2013; Accepted Nov. 10, 2013; Revised Nov. 21, 2013
}

producing animals (Allen et al., 2013). Among them, AMPs are peptides that are generated by the innate immune system and confer specific innate immunity against microbes. Various organisms, including microbes, plants and animals, produce AMPs as the first line of defense to fight against invading pathogenic microbes. AMPs possess broadspectrum of bactericidal activity against Gram-positive bacteria, Gram-negative bacteria, fungi, parasites, virus and tumor cells (Bals, 2000). One of interesting features of AMPs is that they rarely elicit the resistance of bacteria that is a serious problem with conventional antibiotics (Lee et al., 1997). Therefore, AMPs have emerged as one of the most promising candidates for a new class of antibiotics (Hancock and Scott, 2000; Reddy et al., 2004).

Among AMPs, cecropins (Cec) are small ( 4 kDa) peptides containing 35 to 39 amino acids, which are amphipathic, as a high proportion of basic amino acids are present at the $\mathrm{N}$-terminus conferring a net positive charge and the hydrophobic amino acids are rich at the C-terminus (Sipos et al., 1992). Structural analyses revealed the formation of $\alpha$-helix, which is split by a Glycine-Proline 
sequence in the well-studied cecropin A from $H$. cecropia (Steiner, 1982) and cecropin B2 from B. mori (Gazit et al., 1994). To date, four cecropins, namely cecropin P1-P4, had been reported in Ascaris suum which is a parasite found in gut of pig (Pillai et al., 2005). All of them consist of 31 amino acids forming $\alpha$-helix throughout the entire structure and disc diffusion assay using synthetic Cec peptides showed broad-spectrum of antibacterial activity against various pathogenic bacteria (Pillai et al., 2005). These findings suggest that $\mathrm{Cec}$ might be highly potent and effective novel antibiotics. But the cost of producing CecPs by using solid phase chemical synthesis could restrict their practical use as alternatives for antibiotics. Therefore, production of antimicrobial peptides using a microorganism could be an efficient alternative. In this regard, Pichia pastoris, which is a methylotrophic yeast, can be an attractive host for the expression of recombinant protein, as it has been developed as an excellent host for the high level expression of heterologous proteins under the $A O X 1$ (alcohol oxidase1) gene promoter which is tightly regulated by methanol (Cereghino and Cregg, 2000; Cregg et al., 2000; Macauley-Patrick et al., 2005). Therefore, we sought to develop Pichia pastoris expression system to express recombinant $\mathrm{CecP} 4$ and test its antimicrobial activity against pathogenic bacteria for livestocks. The present study shows the successful manipulation of CecP4 for expression in Pichia pastoris and the secretion of a functional AMP to inhibit pathogenic bacteria which may impact livestock health.

\section{MATERIALS AND METHODS}

\section{Cloning of CecP4 cDNA}

To get the CecP4 cDNA, we used RT-PCR technique using isolated total RNA from Ascaris suum. Extraction of total RNA was conducted by using the TRI reagent according to the manufacturer's instruction. Isolated total RNA (500 ng) was reverse transcribed using $5 \mathrm{U}$ of AMV reverse transcriptase $\mathrm{XL}$ for $30 \mathrm{~min}$ at $42^{\circ} \mathrm{C}$. The prepared cDNA was amplified by PCR using the following primers: CecP4-1S, 5'- GCGCGCGAATTCCTACTCGTCATGTTT GC -3'; CecP4-1AS, 5'- GCGCGCTCTAGATATAATTGTT CCCGTAC -3'. The PCR condition was as follows; one cycle of $60 \mathrm{~s}$ at $94^{\circ} \mathrm{C}, 35$ cycles each for $30 \mathrm{~s}$ at $94^{\circ} \mathrm{C}, 30 \mathrm{~s}$ at $58^{\circ} \mathrm{C}$ and $30 \mathrm{~s}$ at $72^{\circ} \mathrm{C}$, and one cycle of $10 \mathrm{~min}$ at $72^{\circ} \mathrm{C}$. PCR products were cloned into pGEM-T (Promega, WI, USA) vector and the sequence was verified.

\section{Subcloning into pPICZB vector and screening}

The PCR product of the full length ORF of Ascaris CecP4 was cleaved with EcoRI/XbaI restriction enzymes (NEB, USA) and was cloned into the $P$. pastoris expression vector pPICZB of EasySelect Pichia Expression Kit
(Invitrogen, CA, USA). The resulting vector (pPICZB::CecP4) contained the full length ORF of CecP4 at downstream of the secretion signal sequence of Saccharomyces cerevisiae $\alpha$-factor prepropeptide and ZeocinTM resistance gene as a selection marker. In order to integrate the pPICZB::CecP4 plasmid into the 5'-AOX1 region of $P$. pastoris (X33), we linearized the pPICZB::CecPs with SacI restriction enzyme (NEB). Transformation was performed by the lithium chloride method according to the manufacturer's protocols (Invitrogen, USA). To determine whether the pPICZB::CecP4 plasmid integrated into $P$. pastoris, individual colonies were picked and analyze for the presence of pPICZB::CecP4 plasmid as well as a control transformant $(\mathrm{C})$ with empty vector (pPICZB). After the genomic DNAs were isolated by the manufacturer's protocols (Invitrogen), the presence of the integrated $\mathrm{CecP} 4$ was confirmed by PCR using the specific primers of $A O X 1$ gene: forward primer; 5'-GACTGGTTCCAATTGACAA GC-3'/ reverse primer; 5'-GCAAATGGCATTCTGACA TCC-3'.

\section{Induction of recombinant CecP4 peptide using methanol}

To determine if the transformed cells were able to secrete the recombinant $\mathrm{CecP} 4$ as an active form with antibacterial activity, the transformants and control (C) were grown in $20 \mathrm{~mL}$ of MM (Minimal methanol; $1.34 \%$ yeast nitrogen base (YNB), $1 \%$ methanol, $400 \mu \mathrm{g} / \mathrm{L}$ biotin) media at $30^{\circ} \mathrm{C}$ for $96 \mathrm{~h}$. The cell culture supernatant was collected by removing the pellet after centrifuging at $500 \mathrm{~g} \mathrm{rpm}$ for 5 $\min$.

\section{Disc diffusion assay}

The supernatants which were concentrated after methanol induction were subjected to the antibacterial activity assay by determining the size of clear zones, i.e., growth inhibition zone against the lawn of bacterial strains tested; Staphylococcus aureus KCTC 1621, Pseudomonas aeruginosa and Escherichia coli, respectively. These bacteria were either purchased from the KRIBB Gene Bank or kindly provided by Dr. Choi Kang Duk at Han-Kyong Nat'l University. Bacterial culture was grown to early log phase (OD at $600 \mathrm{~nm} \approx 0.5$, corresponding to $1 \sim 2 \times 10^{8} / \mathrm{mL}$ $\mathrm{CFU}$ ) and then poured on the test media, followed by placing a cylindrical plastic tube $(6 \mathrm{~mm}$ inner diameter $\times 8$ $\mathrm{mm}$ high) on top of the lawn of bacteria culture. Thereafter, $180 \mu \mathrm{L}$ of the supernatant was loaded inside the tube. The plates were incubated at $37^{\circ} \mathrm{C}$ overnight and the size of the clear zone around each cylindrical tube was observed.

\section{RESULTS}

Cloning of Ascaris CecP4 cDNA into pPICZB P. pastoris 


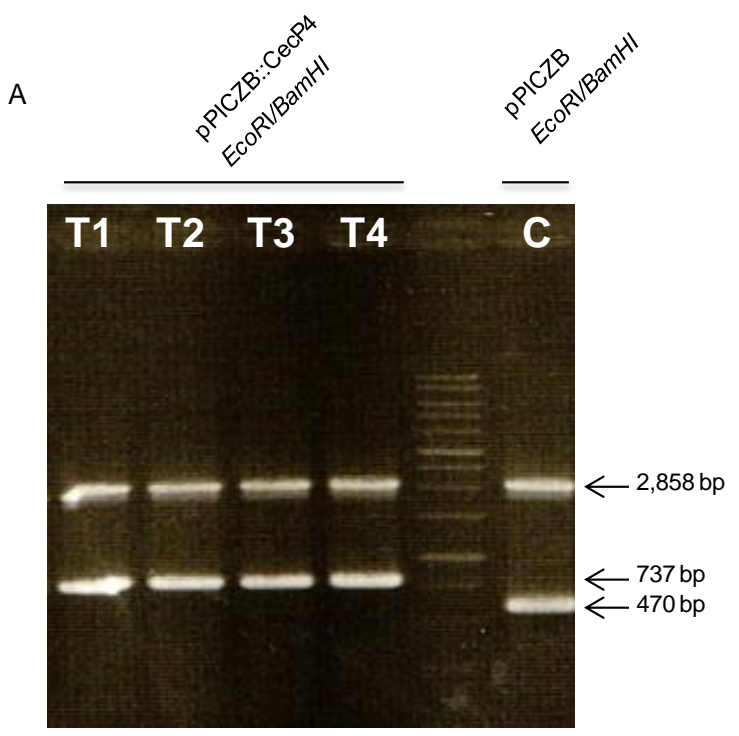

B

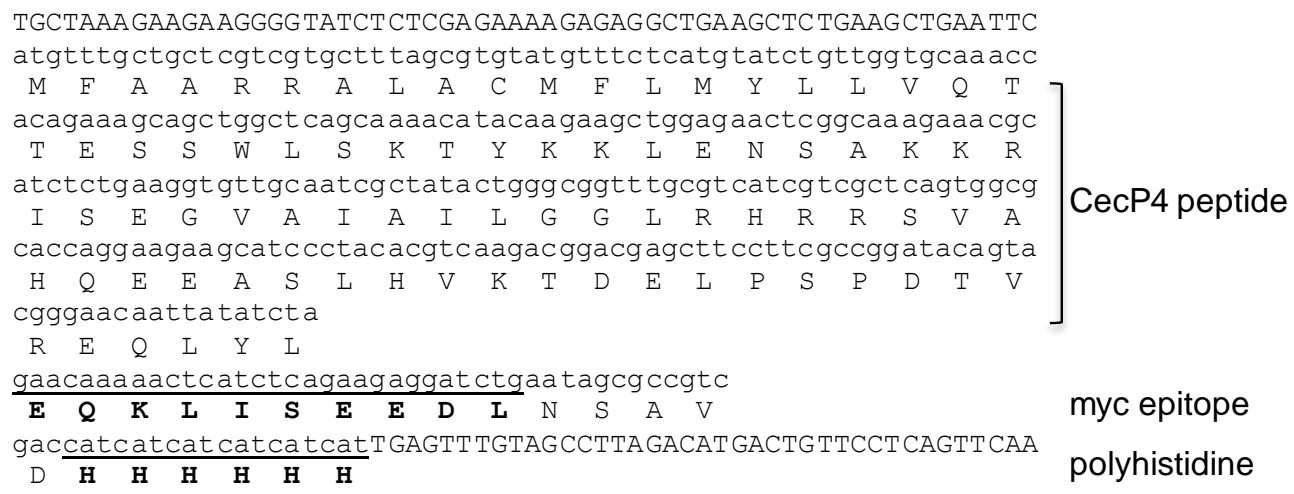

TGCTAAA GAAGAAGGGG TATCT CTCGA GAAAA GAGAG GCTGAAGCTC TGAAG CTGAA TTC atgtttgctgctcgtcgtgctttagcgtgtatgtttctcatgtatctgttggtgcaaacC $\begin{array}{llllllllllllllllllll}M & F & A & A & R & R & A & L & A & C & M & F & L & M & Y & L & L & V & Q & T\end{array}$ acagaaagcagctgget cagcaaaaca tacaagaagctggagaactcggcaa agaaa cgc $\begin{array}{llllllllllllllllllll}T & E & S & S & W & L & S & K & T & Y & K & K & L & E & N & S & A & K & K & R\end{array}$ atctctgaaggtgttgcaatcgctatactgggcggtt tgcgtcatcg tcgctcagtggcg

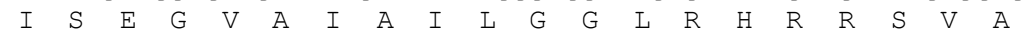
caccaggaagaagcatccctacacgtcaagacggacgagcttccttcgccggatacagta $\begin{array}{llllllllllllllllllll}\mathrm{H} & \mathrm{Q} & \mathrm{E} & \mathrm{E} & \mathrm{A} & \mathrm{S} & \mathrm{L} & \mathrm{H} & \mathrm{V} & \mathrm{K} & \mathrm{T} & \mathrm{D} & \mathrm{E} & \mathrm{L} & \mathrm{P} & \mathrm{S} & \mathrm{P} & \mathrm{D} & \mathrm{T} & \mathrm{V}\end{array}$ cgggaacaattatatcta

myc epitope

polyhistidine

Figure 1. Identification of the integrated pPICZA::CecP4 recombinant plasmid into Pichia pastoris AOX1 gene. (A) Identification of integrated pPICZA::CecP4 by restriction enzyme digestion. Genomic DNAs were isolated from control strain with empty vector, pPICZA (C) and four transformants with pPICZA::CecP4 (T1, T2, T3, and T4) and used for restriction enzyme digestion with EcoRI and $\mathrm{X} b a$ I. (B) Sequences analysis of the insert CecP4 in pPICZA::CecP4. Amino acids with bold letters represent myc epitope and polyhistidine, respectively.

\section{expression vector and screening}

The 267 bp fragment of full length CecP4 ORF cloned into pPICZ B vector $(267 \mathrm{bp}+3,329 \mathrm{bp}=3,596 \mathrm{bp})$ which is verified by restriction enzyme (Figure $1 \mathrm{~A}$ ) and sequencing. The expected expression of the peptide is shown in Figure $1 \mathrm{~B}$. Once we integrated the vector into the 5'-AOX1 region of Pichia pastoris (X33) and confirmed it by PCR, we try to find transformants which has antibiotic activity. Among the many clones, clone \#2 (CecP4-2) showed strong antibacterial activity against Staphylococcus aureus KCTC 1621. After then, we used it for further study.

\section{Recombinant Ascaris CecP4 peptide expression condition}

We investigated several different methanol concentrations to establish the culture conditions for the highest production of CecP4. The clone CecP4-2 was cultured in $20 \mathrm{~mL}$ of $\mathrm{MMY}$ media at $30^{\circ} \mathrm{C}, 200 \mathrm{rpm}$ for 120 h. Different volumes of $100 \%$ methanol were added into the cultures to a final concentration of $1 \%$ and $2 \%$ every day. Samples were harvested every $24 \mathrm{~h}$ and the antibacterial activity of CecP4 was determined by agar diffusion assay. As shown in Figure 2A, the growth rates of the control strain (empty vector) and transformant (CecP4-2) were dependent on methanol concentration of the culture media. The growth of the transformant was higher in $1 \%$ methanol than in $2 \%$ at $48 \mathrm{~h}$ (Figure 2B). The antibacterial activity of the CecP4-2 reached up to its maximum after $72 \mathrm{~h}$ incubation with $2 \%$ methanol, maintaining activity to $120 \mathrm{~h}$, and to a lesser extent at the $72 \mathrm{~h}$ and $96 \mathrm{~h}$ time points with $1 \%$ methanol concentration (Figure 2B). Based on the results, the antibacterial activity of recombinant $\mathrm{CecP} 4$ seems to be correlated with the growth of the strain, but not directly proportional. 

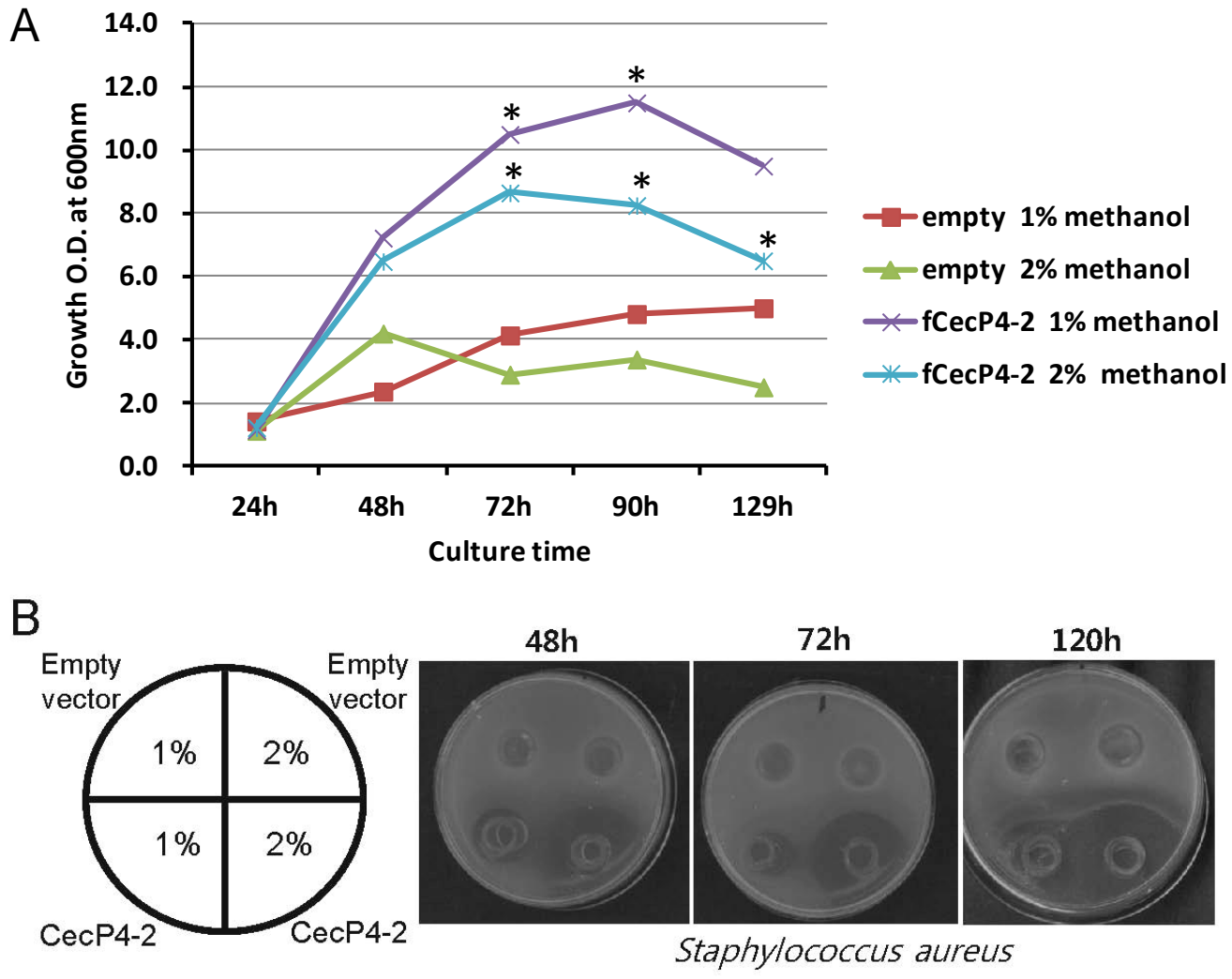

Figure 2. Growth curve of the CecP4 transformant and antibacterial activity of secreted recombinant CecP4 from P. pastoris. (A) Growth curve of $\mathrm{CecP} 4$ transformant with different dose of methanol for induction. For these experiments, CecP4 transformant (CecP4-2) and control transformant were grown in different concentrations of methanol media ( $1 \%$ and $2 \%$ ) for the indicated time before measuring the cell growth by spectrometric analysis of the culture density. This is a representative result of two experiments $(\mathrm{n}=2, * \mathrm{p}<0.05)$. (B) Antibacterial activity of secreted recombinant CecP4 protein against Staphylococcus aureus KCTC 1621. Antibacterial activity was analyzed by agar diffusion assay using the culture supernatants for the indicated time points.

Antibacterial activity of secreted recombinant CecP4 on pathogenic bacteria

We found that concentrated supernatant of recombinant CecP4 maintained bactericidal activity toward Staphylococcus aureus (Figure 3A). In addition, when tested against other pathogenic microbes, i.e., Pseudomonas aeruginosa and Esherichia coli, concentrated recombinant CecP4 exhibited comparable antibacterial activity with gentamycin (Pseudomonas aeruginosa: Figure 3B, Esherichia coli: Figure 3C, respectively), demonstrating that recombinant $\mathrm{CecP} 4$ produced from Pichia pastoris might possess the bactericidal activity toward broad spectrum of pathogenic microbes. Further study warrants to apply Pichia pastoris produced recombinant CecP4 toward other pathogenic microbes.

\section{DISCUSSION}

Cecropin is the antimicrobial peptide family which was initially found in pig intestine (Lee et al., 1989), but later it turned out that $\mathrm{CecP} 1$ and other related family peptides, i.e., $\mathrm{CecP} 2, \mathrm{CecP} 3$, and $\mathrm{CecP} 4$, were expressed in pig intestinal parasite Aascaris suum, more specifically body wall, uterus, ovary, and intestine in response to bacterial infection (Pillai et al., 2005). Screening in the pig and mammalian cDNA libraries failed to identify the homologous sequences to CecPs, suggesting that CecPs were present exclusively in A. suum genome (Philla et al., 2005). All CecPs were bactericidal against Gram-positive such as Staplyococcus aureus, Bacillus subtilis, Micrococcus luteus, and Gramnegative bacteria, including Pesudomonas aeruginosa, Salmonella typhimurium and E. coli (Pillai et al., 2005).

The expression of secreted recombinant proteins in Pichia pastoris offers several advantages over bacterial expression systems. These include appropriate folding of molecules and disulfide bond formation, as well as execution of post-translational modifications which conserve protein function. Secretion of recombinant proteins circumvents intracellular accumulation, a significant aspect in the expression of toxic proteins. The secretion of recombinant proteins also simplifies their purification by avoiding contamination with intracellular proteins (Damasceno et al., 2004; Daly and Hearn, 2005). These advantages make secreted recombinant protein 

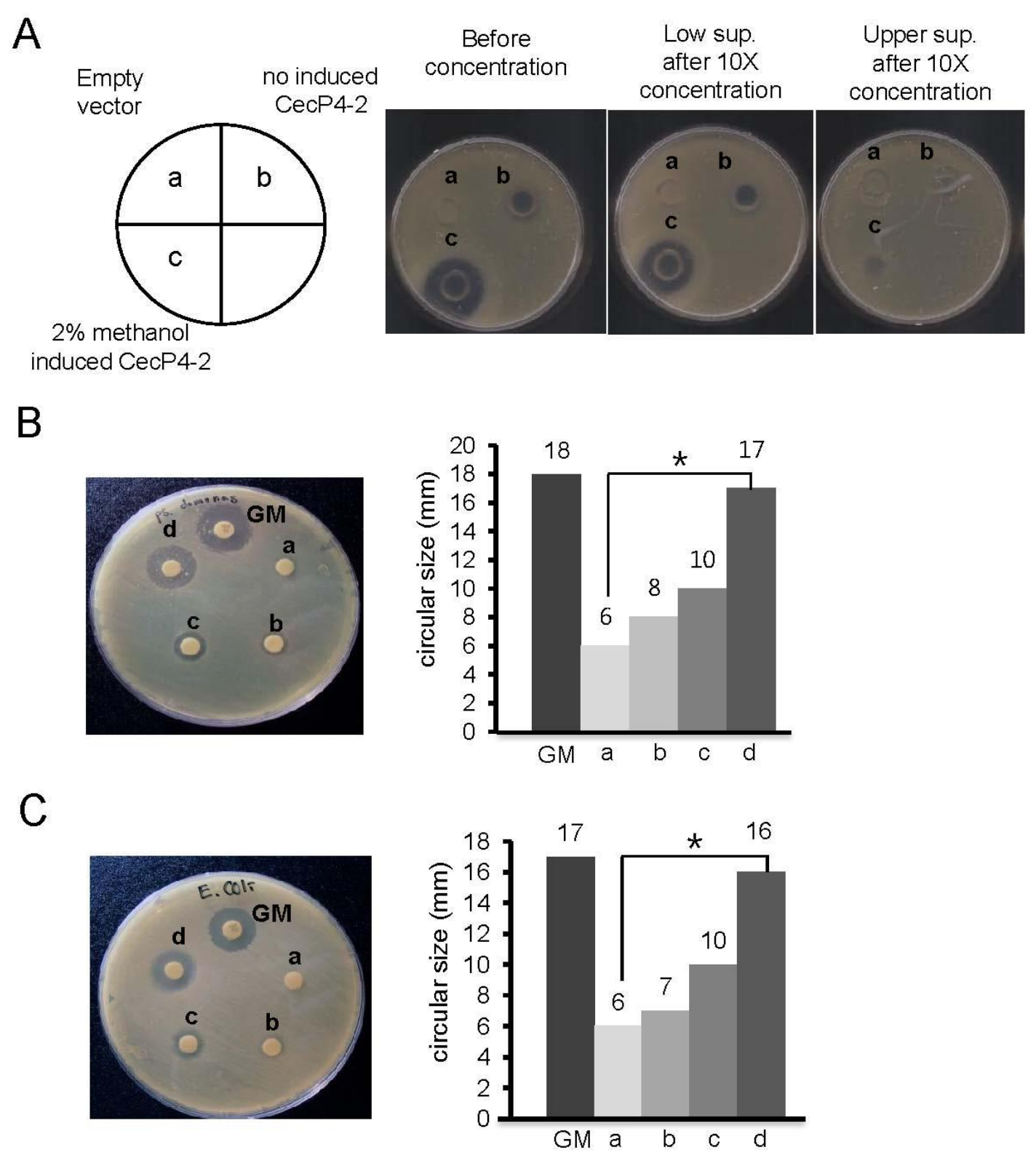

Figure 3. Antibacterial activity of concentrated recombinant CecP4 secreted from P. pastoris using disc diffusion assay. (A) Antibacterial activity of concentrated recombinant CecP4 peptide against $S$. aureus. Culture supernatants were concentrated and upper and lower supernatant after concentration were used for disc diffusion assay, respectively. a: empty vector, b: supernatant (lower), c: supernatant (upper) (This is a representative result of three experiments $(n=3, * \mathrm{p}<0.05)$. (B) Antibacterial activity of concentrated recombinant $\mathrm{CecP} 4$ on Pseudomonas. Pseudomonas were treated with different dose of concentrated recombinant CecP4 and incubated $37^{\circ} \mathrm{C}$ for $18 \mathrm{~h}$. This is a representative result of three experiments $(n=3, * p<0.05)$. Concentrated supernatant of recombinant CecP4 $(10 X)$, a: $25 \mu \mathrm{L}, \mathrm{b}$ : $50 \mu \mathrm{L}$, c: $100 \mu \mathrm{L}$, d: $200 \mu \mathrm{L}$. GM: gentamycin $10 \mu \mathrm{L}(10 \mathrm{mg} / \mathrm{mL})$. (C) Antibacterial activity of concentrated recombinant CecP4 on Escherichia coli. Escherichia coli were treated with different dose of concentrated recombinant CecP4 and incubated $37^{\circ} \mathrm{C}$ for $18 \mathrm{~h}$. This is a representative result of three experiments $\left(\mathrm{n}=3,{ }^{*} \mathrm{p}<0.05\right)$. Concentrated supernatant of recombinant CecP4 $(10 \times)$, a: $25 \mu \mathrm{L}, \mathrm{b}: 50$ $\mu \mathrm{L}$, c: $100 \mu \mathrm{L}, \mathrm{d}: 200 \mu \mathrm{L}$. GM: gentamycin $10 \mu \mathrm{L}(10 \mathrm{mg} / \mathrm{mL})$.

production in Pichia pastoris popular for scientific research (Guo et al., 2012).

In conclusion, the present study demonstrates the successful manipulation of CecP4 for expression in yeast and the secretion of a functional AMP. Further studies are warranted to optimize the culture condition for mass production of recombinant $\mathrm{CecP} 4$ and to test antimicrobial activity against broad spectrum of pathogens from chicken and pigs for the practical application of secreted recombinant CecP4 to livestock production as a feed supplement. 


\section{ACKNOWLEDGEMENT}

This work was supported by the Next Generation of BioGreen Project 21 (PJ0008196), RDA, Republic of Korea and Inha University Research Grant.

\section{REFERENCES}

Allen, H. K., U. Y. Levine, T. Looft, M. Bandrick, and T. A. Casey. 2013. Treatment, promotion, commotion: antibiotic alternatives in food-producing animals. Trends Microbiol. 21:14-119.

Bals, R. 2000. Epithelial antimicrobial peptides in host defense against infection. Respir. Res. 1:141-150.

Cereghino, J. L. and J. M. Cregg. 2000. Heterologous protein expression in the methylotrophic yeast Pichia pastoris. FEMS Microbiol. Rev. 24:45-66.

Cregg, J. M., J. L. Cereghino, J. Shi, and D. R. Higgins. 2000. Recombinant protein expression in Pichia pastoris. Mol. Biotechnol. 16:23-52.

Daly, R. and M. T. Hearn. 2005. Expression of heterologous proteins in Pichia pastoris: a useful experimental tool in protein engineering and production. J. Mol. Recognit. 18:119138.

Damasceno, L. M., I. Pla, H. J. Chang, L. Cohen, G. Ritter, L. J. Old, and C. A. Batt. 2004. An optimized fermentation process for high-level production of a single-chain Fv antibody fragment in Pichia pastoris. Protein Expr. Purif. 37:18-26.

Gazit, E., W. J. Lee, P. T. Brey, and Y. Shai. 1994. Mode of action of the antibacterial cecropin B2: a spectrofluorometric study. Biochemistry 33:10681-10692.

Guo, C., Y. Huang, H. Zheng, L. Tang, J. He, L. Xiang, D. Liu, and H. Jiang. 2012. Secretion and activity of antimicrobial peptide cecropin D expressed in Pichia pastoris. Exp. Ther. Med. 4:1063-1068.
Hancock, R. E. and M. G. Scott. 2000. The role of antimicrobial peptides in animal defenses. Proc. Natl. Acad. Sci. USA. 97:8856-8861.

Lee, J. -Y., A. Boman, C. X. Sun, M. Andersson, H. Jörnvall, V. Mutt, and H. G. Boman. 1989. Antibacterial peptides from pig intestine: isolation of a mammalian cecropin. Proc. Natl. Acad. Sci. USA. 86:9159-9162.

Lee, D. G., J. H. Park, S. Y. Shin, S. G. Lee, M. K. Lee, K. L. Kim, and K. S. Hahm. 1997. Design of novel analogue peptides with potent fungicidal but low hemolytic activity based on the cecropin A-melittin hybrid structure. Biochem. Mol. Biol. Int. 43:489-498.

Macauley-Patrick, S., M. L. Fazenda, B. McNeil, and L. M. Harvey. 2005. Heterologous protein production using the Pichia pastoris expression system. Yeast 22:249-270.

Pillai, A., S. Ueno, H. Zhang, J. M. Lee, and Y. Kato. 2005. Cecropin P1 and novel nematode cecropins: a bacteriainducible antimicrobial peptide family in the nematode Ascaris suum. Biochem. J. 390:207-214.

Reddy, K. V., R. D. Yedery, and C. Aranha. 2004. Antimicrobial peptides: premises and promises. Int. J. Antimicrob. Agents 24:536-547.

Sipos, D., M. Andersson, and A. Ehrenberg. 1992. The structure of the mammalian antibacterial peptide cecropin P1 in solution, determined by proton-NMR. Eur. J. Biochem. 209:163-169.

Steiner, H. 1982. Secondary structure of the cecropins: antibacterial peptides from the moth Hyalophora cecropia. FEBS Lett. 137:283-287.

Ueno, S., K. Kusaka, Y. Tamada, M. Minaba, H. Zhang, P. C. Wang, and Y. Kato. 2008. Anionic C-terminal proregion of nematode antimicrobial peptide cecropin $\mathrm{P} 4$ precursor inhibits antimicrobial activity of the mature peptide. Biosci. Biotechnol. Biochem. 72:3281-3284. 